\title{
Search for Exotic Decays with NA62
}

\author{
Jan Jerhot ${ }^{* \dagger}$ \\ Charles University, Institute of Particle and Nuclear Physics, Prague \\ E-mail: jan.jerhotecern.ch
}

Fixed-target experiments present a complementary tool for studies of the beyond Standard Model physics in either direct searches for weakly coupled states in $\mathcal{O}\left(10^{7}\right)-\mathcal{O}\left(10^{9}\right) \mathrm{eV} / \mathrm{c}^{2}$ mass scale or indirect searches for deviations in known processes induced by physics of much higher energy scales. The NA62 experiment dedicated to study of ultra-rare kaon decays is competitive on both these fronts thanks to its immense sensitivity and possibility of data-taking in beam-dump mode, in which statistics of $\mathcal{O}\left(10^{16}\right)$ POT has been collected and another $\mathcal{O}\left(10^{18}\right)$ POT will follow in 2021-2023 run. This proceedings will present the published results, discuss the current status and show the future prospects of searches for exotic particles at NA62, specifically heavy neutral leptons, dark photons and axion-like particles.

XXIX International Symposium on Lepton Photon Interactions at High Energies - LeptonPhoton2019 August 5-10, 2019

Toronto, Canada

\footnotetext{
${ }^{*}$ Speaker.

${ }^{\dagger}$ On behalf of the NA62 Collaboration: R. Aliberti, F. Ambrosino, R. Ammendola, B. Angelucci, A. Antonelli, G. Anzivino, R. Arcidiacono, T. Bache, M. Barbanera, J. Bernhard, A. Biagioni, L. Bician, C. Biino, A. Bizzeti, T. Blazek, B. Bloch-Devaux, V. Bonaiuto, M. Boretto, M. Bragadireanu, D. Britton, F. Brizioli, M. B. Brunetti, D. Bryman, F. Bucci, T. Capussela, J. Carmignani, A. Ceccucci, P. Cenci, V. Cerny, C. Cerri, B. Checcucci, A. Conovaloff, P. Cooper, E. Cortina Gil, M. Corvino, F. Costantini, A. Cotta Ramusino, D. Coward, G. D’Agostini, J. Dainton, P. Dalpiaz, H. Danielsson, N. De Simone, D. Di Filippo, L. Di Lella, N. Doble, B. Dobrich, F. Duval, V. Duk, J. Engelfried, T. Enik, N. Estrada-Tristan, V. Falaleev, R. Fantechi, V. Fascianelli, L. Federici, S. Fedotov, A. Filippi, M. Fiorini, J. Fry, J. Fu, A. Fucci, L. Fulton, E. Gamberini, L. Gatignon, G. Georgiev, S. Ghinescu, A. Gianoli, M. Giorgi, S. Giudici, F. Gonnella, E. Goudzovski, C. Graham, R. Guida, E. Gushchin, F. Hahn, H. Heath, E. B. Holzer, T. Husek, O. Hutanu, D. Hutchcroft, L. Iacobuzio, E. Iacopini, E. Imbergamo, B. Jenninger, J. Jerhot, R. W. Jones, K. Kampf, V. Kekelidze, S. Kholodenko, G. Khoriauli, A. Khotyantsev, A. Kleimenova, A. Korotkova, M. Koval, V. Kozhuharov, Z. Kucerova, Y. Kudenko, J. Kunze, V. Kurochka, V. Kurshetsov, G. Lanfranchi, G. Lamanna, E. Lari, G. Latino, P. Laycock, C. Lazzeroni, M. Lenti, G. Lehmann Miotto, E. Leonardi, P. Lichard, L. Litov, R. Lollini, D. Lomidze, A. Lonardo, P. Lubrano, M. Lupi, N. Lurkin, D. Madigozhin, I. Mannelli, G. Mannocchi, A. Mapelli, F. Marchetto, R. Marchevski, S. Martellotti, P. Massarotti, K. Massri, E. Maurice, M. Medvedeva, A. Mefodev, E. Menichetti, E. Migliore, E. Minucci, M. Mirra, M. Misheva, N. Molokanova, M. Moulson, S. Movchan, M. Napolitano, I. Neri, F. Newson, A. Norton, M. Noy, T. Numao, V. Obraztsov, A. Ostankov, S. Padolski, R. Page, V. Palladino, A. Parenti, C. Parkinson, E. Pedreschi, M. Pepe, M. Perrin-Terrin, L. Peruzzo, P. Petrov, Y. Petrov, F. Petrucci, R. Piandani, M. Piccini, J. Pinzino, I. Polenkevich, L. Pontisso, Yu. Potrebenikov, D. Protopopescu, M. Raggi, A. Romano, P. Rubin, G. Ruggiero, V. Ryjov, A. Salamon, C. Santoni, G. Saracino, F. Sargeni, S. Schuchmann, V. Semenov, A. Sergi, A. Shaikhiev, S. Shkarovskiy, D. Soldi, V. Sugonyaev, M. Sozzi, T. Spadaro, F. Spinella, A. Sturgess, J. Swallow, S. Trilov, P. Valente, B. Velghe, S. Venditti, P. Vicini, R. Volpe, M. Vormstein, H. Wahl, R. Wanke, B. Wrona, O. Yushchenko, M. Zamkovsky, A. Zinchenko.
} 


\section{Introduction}

NA62 is a fixed-target experiment at CERN SPS (north area), which main goal is to measure the ultra-rare decay $K^{+} \rightarrow \pi^{+} v \bar{v}$ with $10 \%$ precision. The decay is highly suppressed with only $\mathcal{B}\left(K^{+} \rightarrow \pi^{+} v \bar{v}\right)=(8.4 \pm 1.0) \times 10^{-11}$ predicted by the Standard Model [1] and hence is very sensitive to possible contributions stemming from a new physics.

The experiment is supplied from SPS with primary $p^{+}$beam of $400 \mathrm{GeV} / c$, which impinges Be target. A set of apertures (TAX) selects $75 \mathrm{GeV} / c$ component of the secondary beam with $750 \mathrm{MHz}$ particle rate and $6 \%$ component of $K^{+} . K^{+}$in the beam are identified by KTAG (70 ps time resolution) and their momenta are determined by Si-pixel beam spectrometer GTK. 3-momenta of decay products of $\mathrm{K}^{+}$decaying in $60 \mathrm{~m}$ long decay region are measured by STRAW tracker and products are further identified: RICH identifies $\pi^{+}$in $15-35 \mathrm{GeV} / c$ momentum range, further hadron ID is given by combination of calorimeters LKr, MUV1, MUV2 and muon ID is provided by MUV3 placed behind iron wall. In order to reach the needed sensitivity a strong photon rejection is required, where forward region is covered by LKr, large angles by LAV stations and small angles are covered by SAC/IRC. The overall experimental time resolution reaches $\mathcal{O}(100)$ ps.

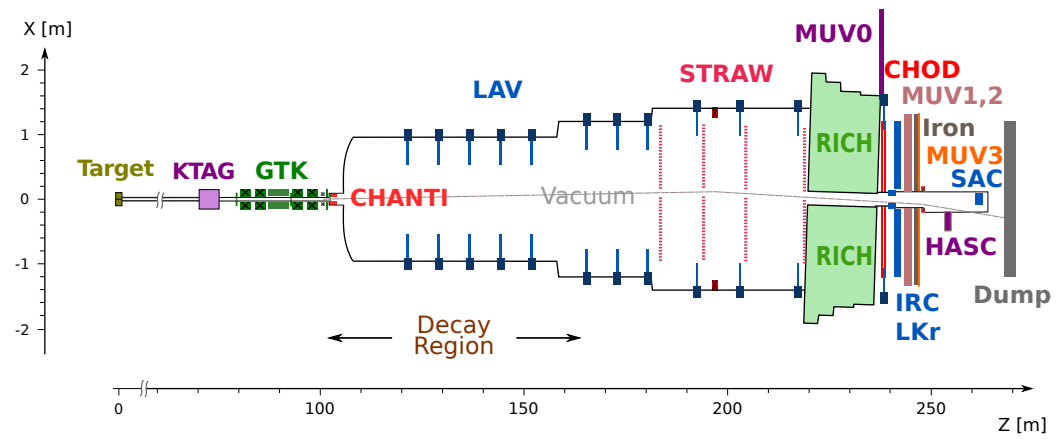

Figure 1: NA62 experimental setup.

The first result on $K^{+} \rightarrow \pi^{+} v \bar{v}$ from the 2016 dataset is published [2], while 2017 and 2018 datasets are being analyzed. The next data-taking period is starting in 2021.

\section{Dark sector searches at NA62}

Thanks to a high energy primary beam, hermetic detector system and time resolution allowing collecting of large statistics NA62 is also competitive in the search for BSM particles (HNL, dark photons, ALPs). Dark sector searches can be performed at NA62 in three different ways:

- $\mathrm{K}^{+}$decays into weakly interacting exotics (bump in missing invariant mass is searched).

- Non-interacting $p^{+}$from primary beam can interact in copper front of the TAX and produce weakly interacting exotic particles. If these particles reach the decay volume and decay out of the main beam they can be recorded using dedicated 'parasitic' trigger running simultaneously with the main kaon trigger.

- 'Beam-dump (BD) mode', where beryllium target is removed and TAX apertures are closed (the primary beam is impinged directly onto TAX). As the beam is effectively ended in TAX a simultaneous operation with other physics modes is not possible. 


\subsection{Dark photons (DP)}

Dark photon $A^{\prime}$ is a gauge boson introduced with an additional $\mathrm{U}(1)$ symmetry and would mix with the SM photon in interaction $\mathcal{L}_{\text {int }} \sim \frac{\varepsilon}{2 \cos \left(\theta_{W}\right)} F_{\mu \nu}^{\prime} B^{\mu v}$, where $\varepsilon$ is the kinetic mixing. Search for DP at NA62 in 'kaon physics mode' in decay chain $K^{+} \rightarrow \pi^{+} \pi^{0}$ and $\pi^{0} \rightarrow \gamma A^{\prime}$ resulted in exclusion shown in figure 2 left [3]. Study of DP production via other meson decays $\left(D, \eta, \eta^{\prime}, \phi\right.$, $\rho, \omega)$ and in Bremsstrahlung-production directly at the Be target can be performed in the future with expected sensitivity as depicted in figure 2 right.
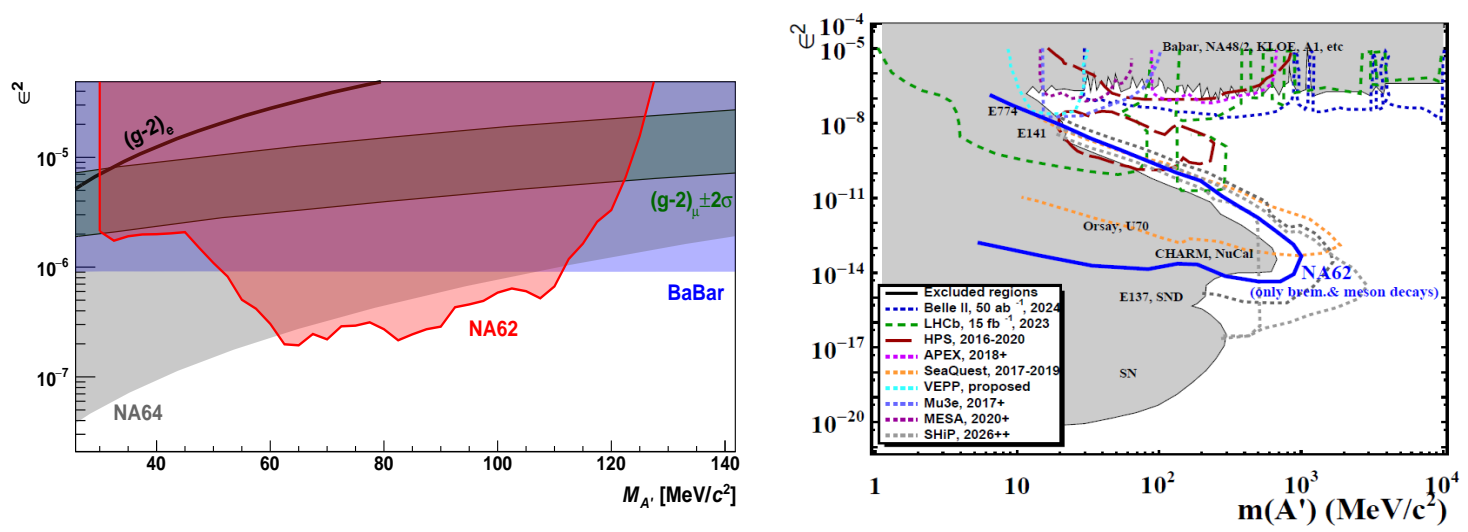

Figure 2: Left: NA62 result for DP exclusion limits (@90\% CL) from decay $\pi^{0} \rightarrow \gamma A^{\prime}$. Right: NA62 prospect on sensitivity (@90\% CL) for DP (based on DP production at Be target for $10^{18}$ POT from meson decays and Bremsstrahlung).

\subsection{Heavy neutral leptons (HNL)}

$v$ MSM introduces 3 additional right-handed neutrinos $N_{i}$ in order to explain the baryon asymmetry and SM neutrino masses, where the lightest $N_{1}$ mass is expected to be $\mathcal{O}(10) \mathrm{keV} / \mathrm{c}^{2}$ (possible DM candidate) and $N_{2,3}$ masses as $\mathcal{O}(1) \mathrm{GeV} / c^{2}$ [4]. Search for HNL in decays $K^{+} \rightarrow \mu^{+} N$ and $K^{+} \rightarrow e^{+} N$ measured during 2015 commissioning run at NA62 led to a new exclusion limit in the $\left(\left|U_{l 4}\right|^{2}\right.$, HNL mass) plane [5] as shown in figure 3. Major improvement (by factor $\mathcal{O}(100)$ ) from 2016-2017 datasets is expected.

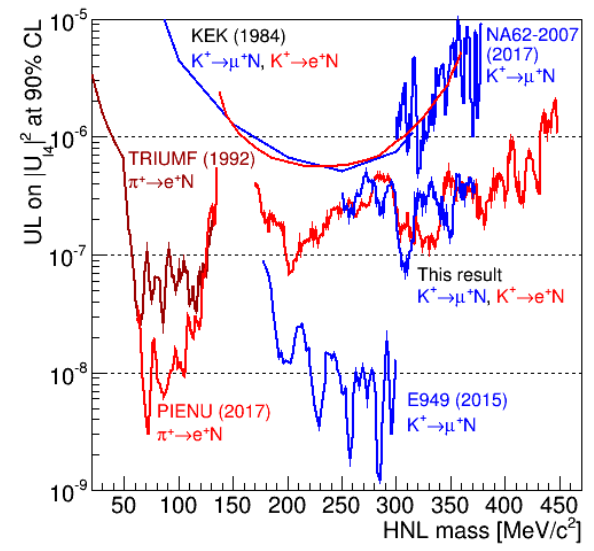

Figure 3: NA62 result ('This result’) for HNL exclusion limits (@90\% CL) from 2015 data sample. 


\subsection{Axion-like particles (ALP)}

ALP is a general pseudoscalar (in most models a pseudo Nambu-Goldstone boson from SSB of an axial U(1) symmetry) with possible coupling to all SM fields. Charged lepton final states (e.g. $A L P \rightarrow \mu \mu$ ) can be searched at NA62 in the 'parasitic mode' run simultaneously with the 'kaon mode'. The common case of predominant coupling to $\gamma$ must be searched in BD mode (no charged particles in the decay volume are allowed), where the dominant ALP production at TAX is via the Primakoff effect with the subsequent decay $A L P \rightarrow \gamma \gamma$ in the decay volume. NA62 is able to probe new regions in the $\left(g_{a \gamma}, m_{a}\right)$ parameter space already with $\sim 10^{16}$ POT $(\sim$ one day of data-taking $)$. The expected NA62 sensitivity for $A L P \rightarrow \gamma \gamma$ with $10^{18}$ POT is depicted in the following figure 4.

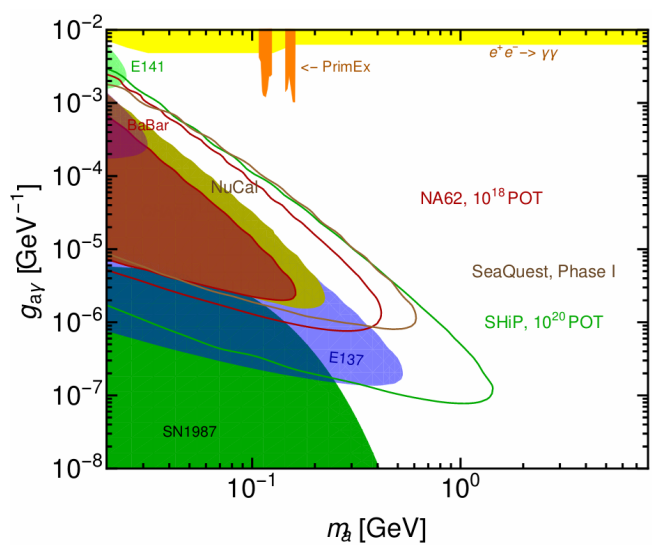

Figure 4: NA62 prospect on sensitivity (@90\% CL) at 10 18 POT ( 3 months of data-taking) in BD mode for ALPs coupled to $2 \gamma[6]$.

\section{Conclusion}

The NA62 experiment has presented the first results on $K^{+} \rightarrow \pi^{+} v \bar{v}$ from the 2016 dataset, while analysis of 2017-2018 data is ongoing and more data will be gathered during 2021-2023 run.

Concerning exotic particles, new limits on coupling and mass were set for DP and HNL. Further improvement is expected with analysis of recent datasets and future data-taking (2021-2023 period) promises even higher sensitivity. NA62 can also search for new particles in a beam-dump mode, where the experiment has a promising sensitivity for axion-like particles.

\section{Acknowledgments}

Support for this work has been received from the grant LTT 17018 of Ministry of Education, Youth and Sports of the Czech Republic and from Charles University Research Center UNCE/SCI/013.

\section{References}

[1] A. Buras et al., JHEP 1511 (2015) 033 [hep-ph/1503.02693].

[2] E. Cortina Gil et al. [NA62 Collaboration], Phys. Lett. B 791 (2019) [hep-ex/1811. 08508 ].

[3] E. Cortina Gil et al. [NA62 Collaboration], JHEP 1905 (2019) 182 [hep-ex/1903 . 08767].

[4] M. Shaposhnikov, JHEP 0808 (2008) 008 [hep-ph / 0804 . 4 542].

[5] E. Cortina Gil et al. [NA62 Collaboration], Phys. Lett. B 778 (2018) [hep-ex/1712 . 0 0297].

[6] B. Döbrich, J. Jaeckel, T. Spadaro, JHEP 1905 (2019) 213 [hep-ph/ 1904.02091 ]. 